\title{
Research on Multi-Objective Optimization of Control Parameters for Switched Reluctance Generators
}

\author{
Lei Dong ${ }^{1 .+}$, Qian Jiang ${ }^{1}$, Lulu Ling ${ }^{1}$, Liwei Shao ${ }^{1}$ \\ ${ }^{1}$ School of Automation, Beijing Institute of Technology, Beijing, P.R.China
}

\begin{abstract}
This paper presents a power generation control parameter optimization model of switched reluctance motor based on differential evolution algorithm. In order to find the solution, the linear weighting method is used to transform the multi-objective optimization problem into a single-objective optimization problem, and then the differential evolution algorithm is used to search the optimal solution of singleobjective optimization. Matlab/Simulink was used to build the multi-objective optimization model of switched reluctance motor to study the effect of the turn-on angle, the freewheeling angle, the turn-off angle on the output power, generation efficiency and DC terminal current ripple under Freewheeling Control Method to find the commutation angle that can make all performance indexes reach the optimal at the same time at different rotational speeds.
\end{abstract}

Keywords: switched reluctance motor, differential evolution algorithm, multi-objective optimization, current ripple

\section{Introduction}

The simple structure without windings and permanent magnets on the rotor, low manufacturing cost and fault tolerance[1-3] make the switched reluctance motor (SRM) a viable candidate for hash environment and high speed applications, such as aerospace[4,5], automotive[6,7] and wind power generation systems[8].

In the traditional Single Pulse Control Method(SPCM), the external winding voltage is only positive and negative. When the rotation speed is not high, the kinematic electromotive force when the switch is turned off may be less than the dc side power supply voltage, resulting in the phase current in the generation phase cannot continue to rise, which reduces the generation efficiency and output power. In order to keep the current rising in the generation stage, the freewheeling technique has been used in [9-12]. In [9], the increased productivity and output power were presented by using an intermediate freewheeling mode in the single pulse control method (SPCM). The freewheeling stage based on PWM control method was introduced in [10], and the optimal set of tum off, turn on and freewheeling angles were determined one by one in order to maximize the energy-conversion efficiency. The freewheeling interval was evenly distributed around the turn off angle in [11] to mitigate the DC bus current ripples, in which case it was believed that the freewheeling technique did not contribute to the output power enhancement. In [12], freewheeling control method (FCM) cannot increase the output power and efficiency, but it can effectively improve the DC terminal current ripple.

Many scholars have done a lot of research on the optimization of control parameters. Literature [13] designed the optimization equation of the turn on and turn off angle based on the rotation speed, and adjusted the reference current through self-adjusting fuzzy control. Literature [14] applies genetic algorithm and neural network to the maximum wind speed tracking based on switched reluctance motor. Genetic algorithm is used to optimize excitation current at different wind speeds to adjust the speed, and then the neural network is trained based on the optimization results, so as to realize online optimization of excitation current.

+ Corresponding author. Tel.: + 86 13910861946;

E-mail address: correspondent_dong@163.com. 
In paper [15], the author established the switched reluctance generator (SRG) model by using the magnetic linkage and torque data obtained by the finite element analysis method, and calculated the optimal turn on angle and turn off angle according to the maximum power corresponding to the given wind turbine speed, so as to maximize the efficiency. The literature [16] proposed a control method to optimize the driving system's performance index torque/ampere by adjusting the turn on angle and the turn off angle online in steady state, adopt EPROM (erasable programmable read-only memory) to realize online adjustment of turn on angle and turn off angle, and established a mathematical model based on least square method to predict and change the corresponding current value of switching angle.

Most scholars optimize the control parameters for a single target, therefore, it is necessary to optimize these performance indicators at the same time under FCM to determine the control parameters.

\section{Liner Analysis of SRG Operation}

Voltage equation for each phase of SRG is given by ${ }^{[17]}$

$$
u_{p h}=R_{p h} i_{p h}+L\left(i_{p h}, \theta\right) \frac{d i_{p h}}{d t}+e_{p h}
$$

Where $u_{p h}$ is the external voltage, $R_{p h}$ is the phase resistance, the phase inductance $\mathrm{L}$ is the function of the phase current $i_{p h}$ and the rotor position $\theta$, and $e_{p h}=i_{p h} \omega d L\left(i_{p h}, \theta\right) / d \theta$ is back electromotive force, where $\omega$ is the angular velocity.

The typical waveforms of the idealized inductance and phase current in conventional SPCM are illustrated in Fig.1 (a). The flux is built up from zero to its peak value, and energy from the DC source and part of the mechanical energy from the prime mover is stored as field energy in the excitation interval $t_{0} \sim t_{3}$. In the interval $t_{3} \sim t_{4}$, the stored field energy is released and mechanical energy is directly converted into electricity energy, and the dc source acquires energy from each phase without any cost when the inductance is minimum. When the inductance is in the positive slope, the rest of the field energy is delivered to the dc source and the prime mover.

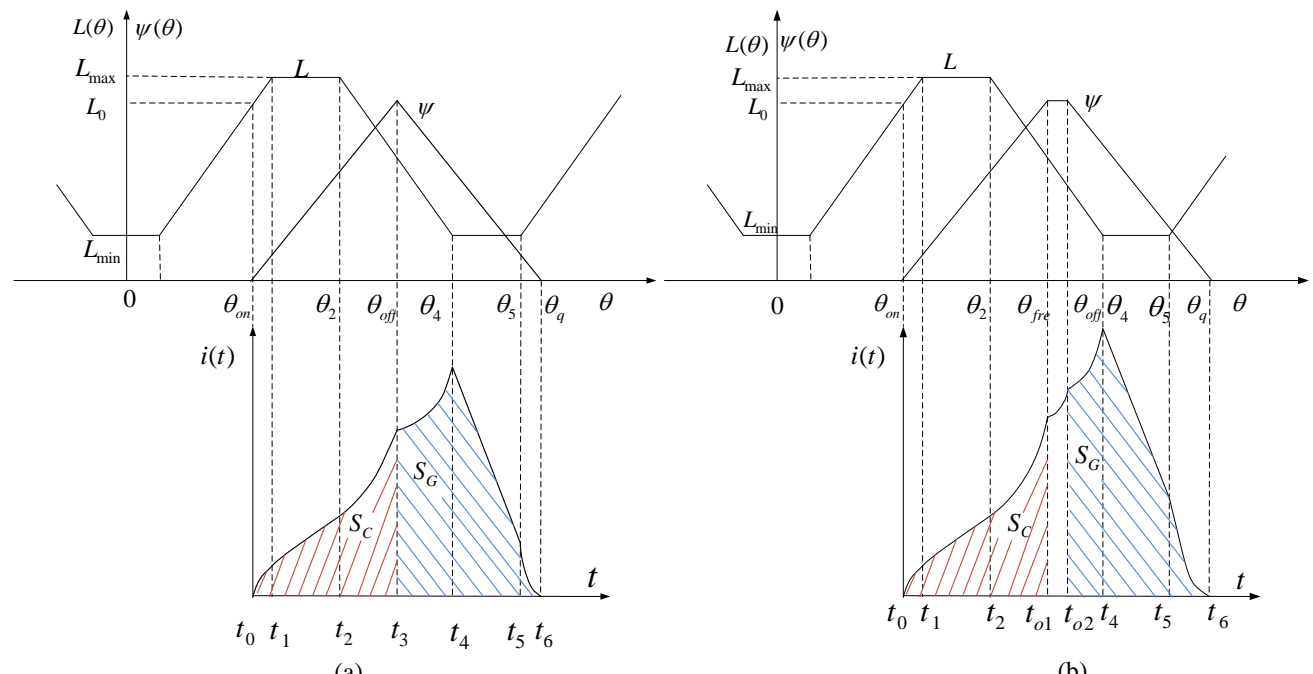

Fig. 1: Typical waveforms of the idealized inductance and phase current: (a) single pulse controlled method; (b) freewheeling controlled method.

In FCM, the freewheeling period, where the phase is short-circuited, is implemented between the excitation interval and the generating interval, and the phase current in FCM is illustrated in Fig.1(b). It can been seen from Fig.1(b) that the phase current keeps rising and the flux maintains constant in the freewheeling interval $t_{o 1} \sim t_{o 2}$, and the mechanical energy is converted into field energy in this period.

The efficiency, torque ripple and DC terminal current ripple coefficients of switched reluctance generators are respectively defined as:

$$
\eta=\frac{P_{\text {out }}}{P_{M}}
$$




$$
\begin{gathered}
T_{e, \text { rip }}=\frac{T_{e, \max }-T_{e, \text { min }}}{T_{e . \text { ave }}} \\
\gamma_{I}[\%]=\frac{\sqrt{I_{d c}^{2}-\left\langle i_{d c}\right\rangle_{\theta=360^{\circ}}^{2}}}{\left|\left\langle i_{d c}\right\rangle_{\theta=360^{\circ}}\right|} \times 100
\end{gathered}
$$

Where, $P_{M}=T_{e . \text { ave }} \omega$ is the average mechanical power output by the prime mover, $\left\langle i_{d c}\right\rangle_{\theta=360^{\circ}}$ represents the average DC terminal current over the entire rotor position cycle, $I_{d c}$ represents the effective value of DC terminal current in the whole rotor position cycle.

\section{Differential Evolution Algorithm}

Differential Evolution (DE) algorithm[18] encodes individuals in a population in real numbers, takes the differences of individuals in each generation of the population as disturbances, reorganizes the disturbances with an individual to get an intermediate population, and then generates a new population through the competition between father and son to promote the evolution of the population.

\subsection{The Basic Principle of DE}

The basic principle of the algorithm is as follows: firstly, the difference vector between the parent generation is used to obtain the variant individual; then the mutant and the parent were crossed to get the test individual; finally, one to one selection operation is adopted to select the better individual between two individuals as the next generation population. The standard differential evolutionary algorithm includes three core operations: mutation, crossover and selection. The following is a detailed analysis of its initialization process and each operator ${ }^{[21]}$ :

1) Initialization

For a population of $N P$ size, if the dimension of the decision variable is $\mathrm{m}$ and $\mathrm{g}$ is the current evolutionary algebra, then the individuals of generation $\mathrm{g}$ can be described as:

$$
X_{i}(\mathrm{~g})=\left[x_{i 1}(\mathrm{~g}), x_{i 2}(\mathrm{~g}), \cdots, x_{i m}(\mathrm{~g})\right] \quad i=1,2, \cdots, N P
$$

where the scope of the entire decision domain is $\left(X_{\text {min }}, X_{\text {max }}\right), X_{\text {min }}=\left\{x_{1, \text { min }}, x_{2, \text { min }}, \cdots, x_{m, \text { min }}\right\}$ and $X_{\text {max }}=\left\{x_{1, \text { max }}, x_{2, \text { max }}, \cdots, x_{m, \text { max }}\right\}$. In order to make the initial population cover the whole decision space as much as possible, $N P$ individuals are generally generated by uniformly distributed random function to form the initial population. For the $j$ th dimension decision variable of the $i$ th individual, the corresponding coding of the initial individual is:

$$
x_{i j, 0}=x_{j, \min }+\operatorname{rand}(0,1) \cdot\left(x_{j, \max }-x_{j, \min }\right)
$$

\section{2) Variation}

For each target individual in the $g$ generation population, randomly selecting two individuals numbered $r 2$ and $r 3$, which have the difference vectors, then randomly selecting an individual numbered $r 1$, and use the difference vector to disturb it to generate variation vector, i.e.

$$
V_{i}(\mathrm{~g})=X_{r 1}(\mathrm{~g})+F \cdot\left[X_{r 2}(\mathrm{~g})-X_{r 3}(\mathrm{~g})\right]
$$

Where, $r 1, r 2$ and $r 3$ are not equal to each other and are not equal to the number $i$ of the target individual, so the population size cannot be less than 4 . Variation factor $F$ is any real number between 0 and 2 , which is used to control the step size of variation individual in the direction of difference vector $\left[X_{r 2}(\mathrm{~g})-X_{r 3}(\mathrm{~g})\right]$, it is usually called expansion factor.

3) Cross

There are two main ways of algorithm crossing: binomial crossing and exponential crossing. For binomial crossover, the first to target individuals in every decision component to generate a uniform distribution on $[0,1]$ between random number randa and randb if random number randa is not greater than 
the crossover probability $C R$ or randb and decision space dimension is equal to the product of the goals of the individual number, with the variation of individual decision component targets individuals for the corresponding component, otherwise, the goal of the individual component is constant expressed in mathematical expressions:

$$
u_{i, j}(\mathrm{~g})= \begin{cases}v_{i, j}(\mathrm{~g}), & \text { if } \text { randa } \leq C R \text { or } j=\text { randb } \cdot m \\ x_{i, j}(\mathrm{~g}), & \text { else }\end{cases}
$$

Where randb is to ensure that at least one one-dimensional component of the test individual is different from the target individual, so that the crossover phenomenon will definitely occur, so as to effectively avoid the population falling into the stagnation of evolution.

4) Selection

Since the variation operation may cause the components in the variation individual to overstep the boundary, after the crossover operation, the nearest boundary value is usually taken to replace the overstepping components or the components are reinitialized to solve the overstepping test individual. The standard differential evolution algorithm uses greedy selection method to retain the excellent individual. The specific selection operation is:

$$
X_{i}(\mathrm{~g}+1)= \begin{cases}V_{i}(\mathrm{~g}), & \text { if } f\left(V_{i}(g)\right) \leq f\left(X_{i}(\mathrm{~g})\right) \\ X_{i}(\mathrm{~g}), & \text { else }\end{cases}
$$

The selection operation makes the individuals of the next generation population better than the individuals of the parent population and promotes the evolution of the population in a good direction. With the improvement of evolutionary algebra, the individuals of the population gradually approach the optimal solution set.

\subsection{Analysis of DE for SRG}

From the basic principle of DE, it can be seen that population size $N P$, number of iterations $G$, variation factor $F$ and crossover probability $C R$ are artificially determined, so different values are selected, and the algorithm requires different running time and final results.

The larger the population size $N P$ is, the larger the number of individuals will be, the better the diversity of the population will be, and it will be easier to carry out variation, and the possibility of getting trapped in local optimization will be reduced, making the optimization ability stronger, but it will increase the calculation amount. Since every iteration will let excellent individuals into the next generation, the more the number of iterations $G$, the more excellent the individuals in the population, that is to say, the optimal solution found will be closer to the Pareto frontier, obviously, the optimization time will be longer. Variation factor $F$ also affects the diversity of population, because it determines the direction and size for each of the variants, $F$ is larger, the greater the degree of variation, the algorithm is global search ability is stronger, but when $F$ is too large, the population will be difficult to convergence, the smaller the $F$, the convergence of algorithm is better, will increase convergence speed is fast, but easy to premature. Practical experience shows that $C R$ controls the possibility of each component of the mutant participating in the crossover operation between 0.5 and 0.9 . The larger $C R$ is, the greater the population variability will be, and the crossover variable is generally set between 0.3 and 0.9 .

\section{Multi-objective Optimization Model of SRG}

FCM has a lot of control parameters, such as the excitation voltage, the phase voltage, the angular velocity, the turn-on angle, the freewheeling angle, the turn-off angle, etc., this paper studies three phase angle change of output power, efficiency, and the influence of the DC side current ripple, found under different angular velocity at the same time make each performance index achieves the optimal in phase angle, then studies the changing relation between the optimal in phase angle with angular velocity.

The dimension of decision variables of SRG power generation control parameters optimization problem $m=3$; the individual of the population is expressed as $X=\left[\theta_{o n}, \theta_{f r e}, \theta_{o f f}\right]$; and the smaller the turn on angle is, the larger the output power will be. However, when the freewheeling angle and turn on angle are larger than 
a certain value, too small turn on angle will reduce the efficiency. When the freewheeling angle and turn off angle are too small, the output power and efficiency are low, but too large will lead the current ripple increase rapidly. Therefore, in order to speed up the running speed of the algorithm, the interval segment that the optimal commutation angle can never appear can be removed, so as to reduce the decision domain. The number of subitems of the problem is $n=3$, and the subitems are $f_{1}(X)=P(X), f_{2}(X)=\eta(X)$ and $f_{3}(X)=\gamma_{I}(X)$, respectively. The constraint condition of the optimization problem is that the freewheeling angle cannot be larger than the turn off angle and the excitation current must not exceed a certain value. Since the phase winding of the motor cannot withstand infinite current, the upper limit of excitation current is selected according to the rated current of the motor. If the excitation current exceeds the set limit, it indicates that the motor is unsafe at this working point, and the motor should not work at this working point.

In order to balance the importance of each objective component in the optimization process and set the same weight for each sub-objective, it is necessary to normalize the value of each sub-objective function. The evaluation function used for the selection operation in DE of this article is:

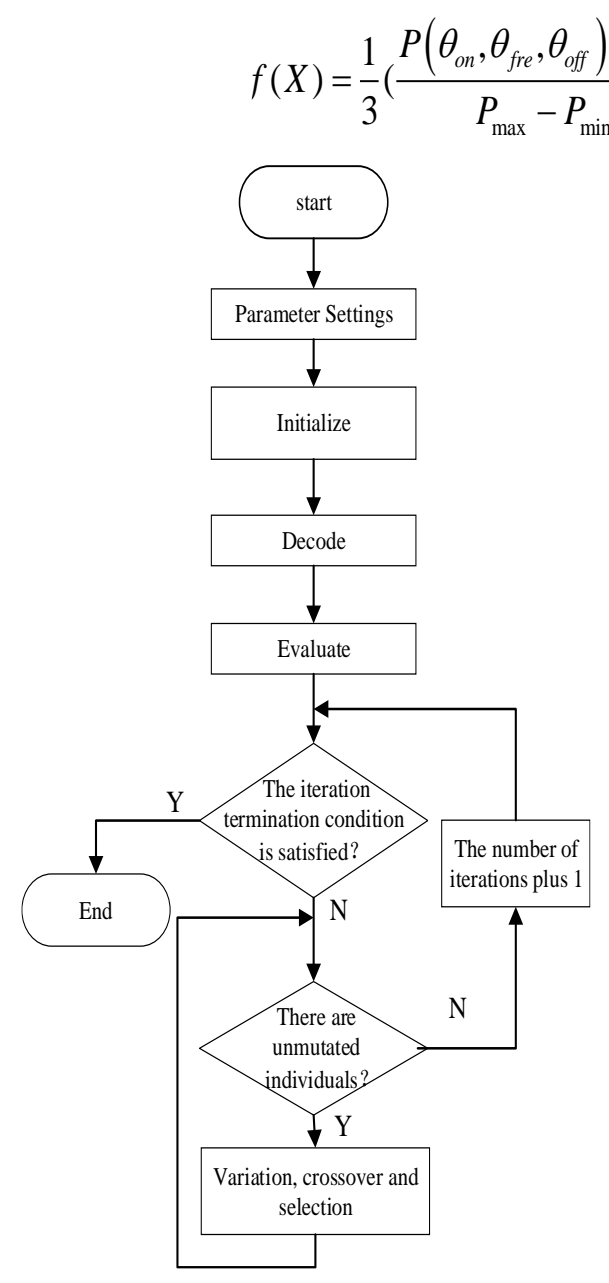

Fig. 2: Optimization program flowchart

Fig. 3: The flow chart of individual adaptive value calculation

The constraint condition that the freewheeling angle cannot be greater than the turn off angle is used as the constraint violation degree. The constraint condition that the excitation current cannot exceed the limit is directly reflected in the evaluation function. If the excitation current exceeds the limit, the value of the evaluation function is set as the worst. The population size is 15 times the dimension of the decision variable, that is, $N P=45$. Variation $\mathrm{F}$ and crossover $C R$ were 0.6 and 0.9 , respectively. The termination condition of $\mathrm{DE}$ in this paper is not that the number of iterations reaches the maximum, but that the difference between the value of the optimal evaluation function and the value of the first 17 generations is less than 0.0001 . The optimization procedure of SRG power generation control parameters is shown in fig. 2 . 
The parameters set in fig2 include those in the differential evolution algorithm (such as the population size of the decision domain maximum iteration number variation and crossover factor), The values of each sub-objective function normalize the required variables(such as the maximum and minimum of output power, efficiency and current ripple coefficient) and parameters used to invoke the Simulink model when evaluating individuals(such as time delay $\Delta t$ and simulation time $T$ ). The output power efficiency and the total objective function of all individuals in the population are initialized to negative infinity, the current ripple coefficient is initialized to positive infinity, and the constraint violation degree is initialized to 0 .

The evaluation and selection of individuals in this optimization program involves the calculation of individual fitness values, and the calculation steps of fitness values of each individual are shown in fig.3.

The entire optimization program is completed in Matlab. When the individual needs to be evaluated, the Simulink model is directly invoked through the sim('name', $T$ ) function for simulation. The Simulink simulation model used in the multi-objective optimization in this paper is shown in fig.4. The output parameters of the Simulink file will be saved to the workspace, providing performance parameters to the optimization program of the $\mathrm{M}$ file.

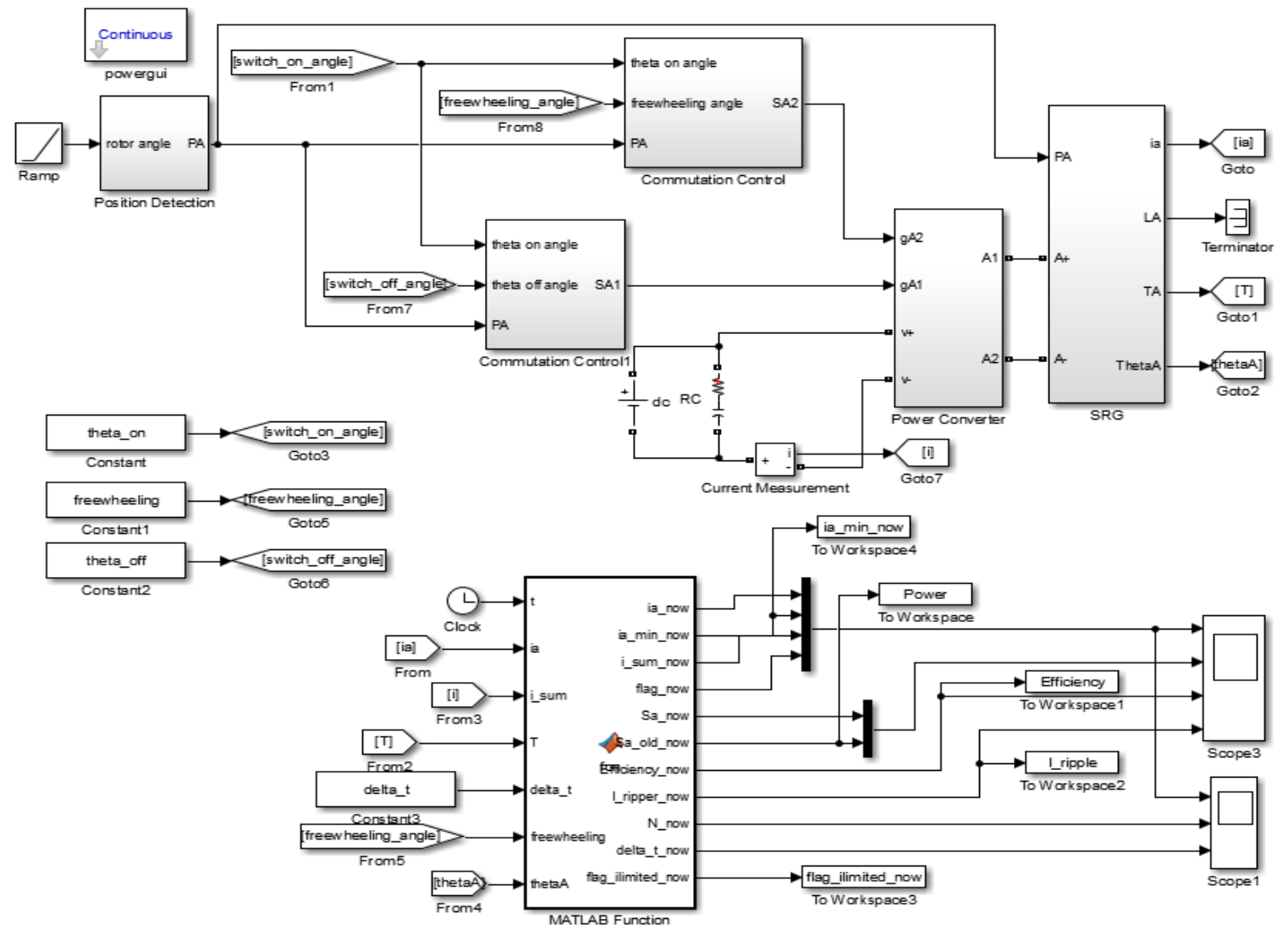

Fig. 4: Multi-objective Optimization Model of SRG in Simulink

\section{Simulation Results and Analysis}

When SRG speed is $1000 \mathrm{rpm}$, excitation voltage and phase voltage of $48 \mathrm{v}$, according to the simulation, scanning discrete working point for rotary speed of the output power, efficiency and the scope of the current ripple coefficient and the optimal value of working point position to make a general evaluation, normalized used to determine the most value.

The variation ranges of turn on angle, freewheeling angle and turn off angle is $4^{\circ} \sim 8^{\circ}, 20.5^{\circ} \sim 25^{\circ}, 21^{\circ} \sim 25.5^{\circ}$, respectively, with an increment of $0.5^{\circ}$. The maximum and minimum output power in this range is $645.8 \mathrm{w}$ and $436.6 \mathrm{w}$, respectively. The efficiency and current ripple coefficients of the optimal power working point are 0.7135 and 2.657 , respectively. The maximum and minimum values of efficiency are respectively 0.8534 and 0.72 , and the maximum and minimum values of current ripple coefficients are respectively 3.0367 and 2.2906 . In this case, the commutation angle pair represented by the optimal individual of the population is $\left(5.695^{\circ}, 21.99^{\circ}, 22.87^{\circ}\right)$. 
In this optimization simulation, two individuals were randomly selected from the population to observe their evaluation function value in the iterative process, as shown in fig.5. The evaluation function value of the optimal individual in each generation of population was recorded, as shown in fig6. Fig7 is the comparison between the performance indexes of the optimal individual in each generation and the optimal power working point. The red black and blue points represent power efficiency and current ripple respectively.

As can be seen from fig.5, the population is always in the state of evolution, and the degree of evolution is different for different individuals. Fig. 6 and fig.7 show that the whole population is evolving towards the optimal evaluation function.

At the end of optimization, the power of the optimal working point was sacrificed by $7.4 \%$, which increased the efficiency by $7.25 \%$ and reduced the current ripple coefficient by $7.8 \%$. After several optimizations, the optimal commutation angle pair at 1000rpm was obtained $(5.6086,21.6348,23.2538)$. The working point only sacrificed $3.118 \%$ of the power, which increased the efficiency by $4.5 \%$ and reduced the current ripple coefficient by $5.46 \%$.

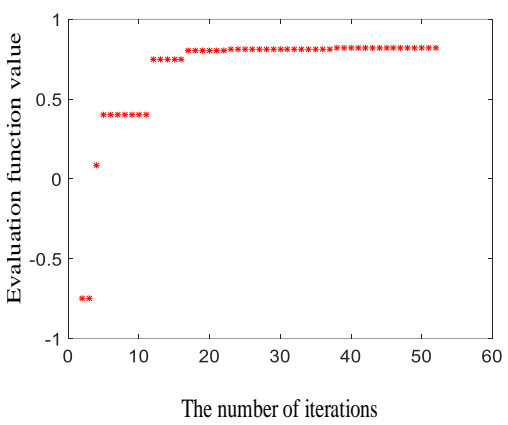

Fig. 5: evaluation function values of two individuals under a certain evaluation function

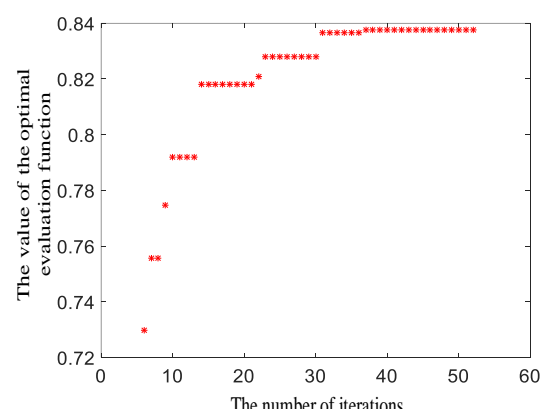

Fig. 6: the value of the optimal evaluation function under an evaluation function

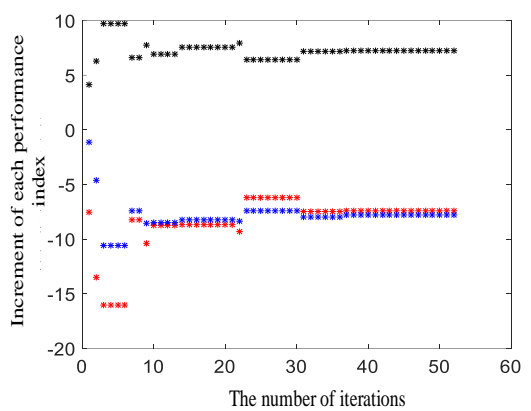

Fig. 7: changes of performance indicators under an evaluation function

Change speed, choose the right most value after normalization processing simulation, and the optimal phase angle change along with the change of speed curve as shown in fig.8. From fig.8, the optimal continuation angle increases with the increase of the rotational speed, and there is no obvious relationship between the optimal turn off angle and the optimal turn on angle and the rotational speed. However, when the rotational speed is larger than a certain value, the variation trend of the two with the rotational speed is basically the same.
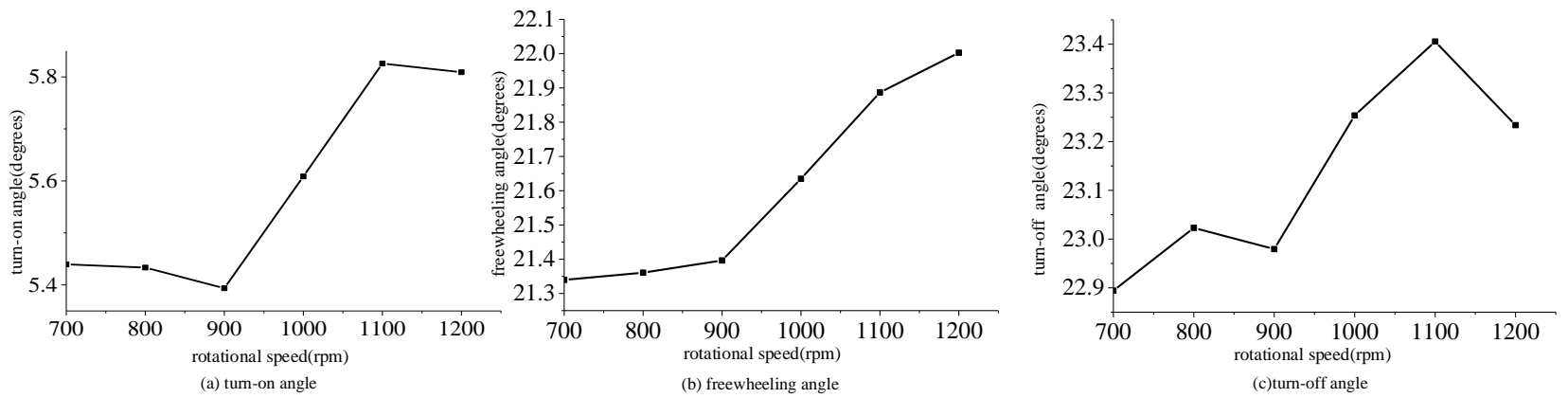

Fig. 8: The relation between optimal commutation angle and rotational speed

\section{Conclusion}

This paper introduces the basic principle of differential evolution algorithm and the influence of various parameters on the optimization results, establishes the multi-objective optimization model of SRG, and finds the output power efficiency of SRG based on the continuation control method at different rotation speeds, as well as the optimal turn on angle, the freewheeling angle and turn off angle of current ripple synthesis.

\section{Acknowledgements}


This work is supported by Guangdong Province Science and Technology Innovation Strategy Special Fund. Project NO. is skjtdzxrwqd2018001.

\section{References}

[1] Cardenas, R., et al. Control of a switched reluctance generator for variable-speed wind energy applications. IEEE Transactions on Energy Conversion 20.4(2005):0-791.

[2] Ding, W., and D. Liang. A Fast Analytical Model for an Integrated Switched Reluctance Starter/Generator. Energy Conversion IEEE Transactions on 25.4(2010):p.948-956.

[3] Vujii, Vladan P., and M. P. Alasan. Simple Sensorless Control for High-Speed Operation of Switched Reluctance Generator. IEEE Transactions on Energy Conversion 31.4(2016):1325-1335.

[4] Radun, and V. A. High-power density switched reluctance motor drive for aerospace applications. IEEE Transactions on Industry Applications 28.1(1992):P.113-119.

[5] M. Tursini, M. Villani, G. Fabri, and L. Di Leonardo, A switched-reluctance motor for aerospace application: Design, analysis and results. Electric Power Systems Research 142.JAN.:74-83.

[6] Raminosoa, T., et al. Design and Optimization of a Switched Reluctance Motor Driving a Compressor for a PEM Fuel-Cell System for Automotive Applications. Industrial Electronics, IEEE Transactions on 57.9(2010):p.29882997.

[7] Multiphysics NVH Modeling: Simulation of a Switched Reluctance Motor for an Electric Vehicle. IEEE Transactions on Industrial Electronics 61.1(2014):469-476.

[8] Tarcio, et al. Approach for performance optimization of switched reluctance generator in variable-speed wind generation system. Renewable Energy (2016).

[9] Dixon, S. , and B. Fahimi. Enhancement of output electric power in switched reluctance generators. IEEE International Electric Machines \& Drives Conference IEEE, 2003.

[10] Kosmatin, P., D. Miljavec, and D. Voncina. Increasing efficiency of the switched reluctance generator at lowspeed operation. International Conference on Compatibility \& Power Electronics IEEE, 2013.

[11] V. Nasirian, S. Kaboli, and A. Davoudi, Output power maximization and optimal symmetric freewheeling excitation for switched reluctance generators, IEEE Trans. Ind. Appl., vol. 49, no. 3, pp. 1031-1042, May.-Jun. 2013.

[12] Ling, Lulu, L. Dong, and X. Liao. Comparison of two control methods of switched reluctance generator. IEEE Conference on Industrial Electronics \& Applications IEEE, 2017.

[13] Zhen-Zhen, Zhou, et al. The Maximum Wind-Energy Tracking Control for Switched Reluctance Generator Based on Integral-Separation Fuzzy PID. small \& special electrical machines (2012).

[14] Zhao, Huang, C. Jiang-Zhou, and W. Jun-Li. Research on MPPT control for SRG wind power generation system. chinese journal of power sources (2016).

[15] Choi, Da Woon, S. I. Byun, and Y. H. Cho. A Study on the Maximum Power Control Method of Switched Reluctance Generator for Wind Turbine. IEEE Transactions on Magnetics 50.1(2014):1-4.

[16] XiLian, Wang, and W. XuDong. Angles optimal control of switched reluctance motor, Electric Machines \& Control 10.6(2006):588-591.

[17] $\mathrm{Hu}, \mathrm{K}$, et al. A Switched-Reluctance Generator With Interleaved Interface DC-DC Converter. Energy Conversion, IEEE Transactions on 30.1(2015):273-284.

[18] Storn, Rainer, and K. Price. Differential Evolution - A Simple and Efficient Heuristic for global Optimization over Continuous Spaces. Journal of Global Optimization 11.4(1997):341-359.

[19] Price, K. V., R. M. Storn, and J. A. Lampinen. Differential Evolution-A Practical Approach to Global Optimization. Natural Computing 141.2(2005). 